\title{
EDITORIAL
}

\section{Long noncoding RNA: noncoding and not coded}

A correction has been published for this paper. The error has been fixed in both the HTML and PDF versions of the paper.

Cell Death Discovery (2017) 3, 16104;

doi:10.1038/cddiscovery.2016.104; published online 9 January 2017

Mammalian evolution exhibits extraordinary acceleration in phenotypic complexity while displaying genetic conservation. Indeed, protein-coding genes are $90 \%$ identical between mice and human, and the question arises what might be the mechanisms by which phenotypic complexity increases while the number of proteins does not? An interesting possibility is that the increase in regulatory complexity, as reflected in a more sophisticated spatiotemporal regulation on gene expression, could generate more diverse combinations of proteins and a greater number of distinct phenotypes. Importantly, unlike protein coding genes, long noncoding RNAs (IncRNA) exhibit only 50\% similarity between mice and human. ${ }^{1}$ Are these RNA species involved in such regulation?

In recent years, IncRNA have captured the attention of biologists. LncRNA are highly abundant in the human genome, and it has been demonstrated that they have fundamental roles in a variety of biological processes. In particular, IncRNA have been shown to participate in gene regulation by binding to proteins, such as chromatin regulators, and targeting them to particular regions in the genome. ${ }^{2,3}$ The interaction between IncRNA and specific proteins often depends on an explicit sequence within the IncRNA, ${ }^{4-6}$ and there has been a surge in new techniques to investigate IncRNA-protein interactions of late. ${ }^{7}$ However, in some cases, it seems that IncRNA-protein interactions are promiscuous and sequence independent. ${ }^{8}$ Furthermore, it has been demonstrated that IncRNA regulate genes in cis and their transcription per se, not the actual sequence, is activating the adjacent gene. ${ }^{9}$ Considering that there are many such IncRNA/protein coding RNA gene pairs in our genome, ${ }^{10}$ it is important to determine the extent and functions of protein coding gene regulation by adjacent IncRNA genes.

Two recent works ${ }^{11,12}$ have demonstrated that a number of IncRNAs act in cis to regulate the transcription of neighboring genes. By analyzing 12 IncRNA loci in mouse embryonic stem cells, Engreitz et al. ${ }^{11}$ found that knocking out the promoter of five of these IncRNAs cis-modulated the expression of nearby genes, which were located within $5-71 \mathrm{~kb}$ from the promoters. To assess whether these cis effects were driven by the IncRNAs sequence, by the transcription process itself, or by DNA regulatory elements present in the promoter, polyadenylation signals were inserted in the studied IncRNA genes to prevent their transcription while preserving the promoter integrity. In a number of cases, the insertion of polyadenylation signals did not impact the transcription of neighboring genes, 11 arguing that in these cases transcription of IncRNAs is not involved in modulating nearby gene expression. These experiments thus support the notion that enhancer-like DNA regulatory elements are present in such IncRNA promoters. In other cases, termination of IncRNA transcription impacted neighboring gene transcription in $\mathrm{cis}^{11,12}$ indicating that the transcription of some IncRNAs is required for the observed cis effect. However, in all cases, specific IncRNA sequences were not required to mediate such effects, as exon deletion or sequence replacement did not affect nearby gene expression. ${ }^{11,12}$ Moreover, while a number of IncRNAs are not conserved, the DNA regulatory elements with enhancer activity present in IncRNA promoters are highly conserved, ${ }^{11}$ highlighting the functional importance of these cis-regulatory elements.

Such cis gene regulatory mechanism is physiologically relevant as shown in mouse models. ${ }^{12}$ Anderson et al. ${ }^{12}$ demonstrated

a
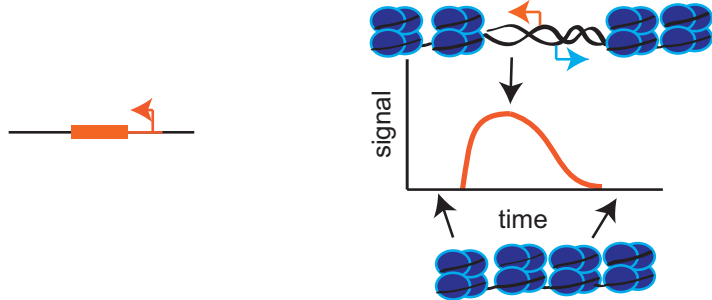

b

cis functions
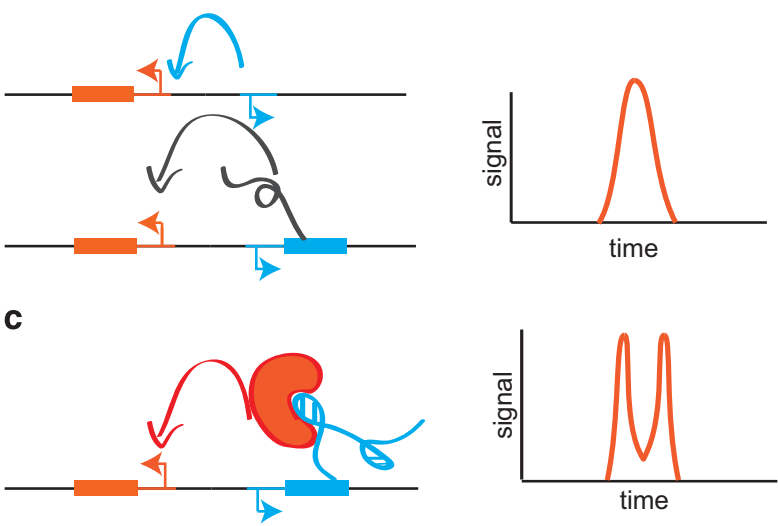

d

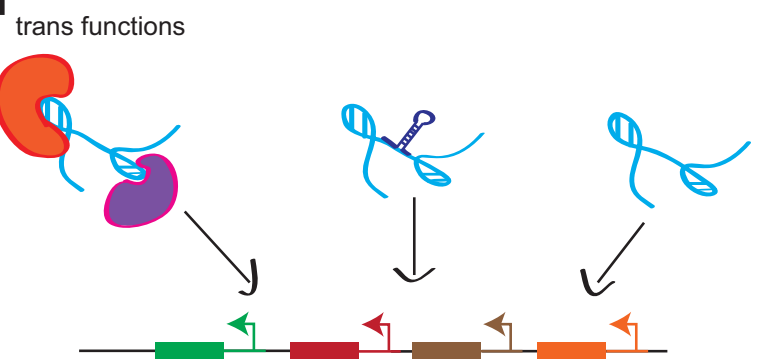

Figure 1. Hierarchy of IncRNA evolution linked to increase complexity of gene regulation. (a) the spatio-temporal regulation of the extent and strength of gene transcription is regulated by the gene's promoter and chromatin state. (b) an adjacent promoter can add another layer of regulation to increase the tightness of the signal by the promoter activity directly and/or by the transcript arising for such a promoter (cis function). (c) the long noncoding transcript may bind proteins to further increase the complexity of regulation of the adjacent protein-coding gene (cis function). (d) the transcript may acquire trans functions such as binding to proteins and regulating their activity, sponging microRNA or binding to chromatin at distal locations. 
in vivo that the transcription of the heart development regulator Hand 2 is controlled in cis by the IncRNA upperhand (Uph), which is located $150 \mathrm{bp}$ distant on the opposite strand. Gene-targeted knockout of Uph, but not knockin of a heterologous DNA sequence into the Uph locus, blocked expression of Hand2 in mouse heart and gave rise to a similar cardiac phenotype as Hand2 knockout embryos. The cis effect is therefore dependent on Uph transcription but not on its sequence. Uph transcription was found to be required to maintain histone marks characteristic of super-enhancers, and to recruit the GATA4 transcription factor to the Hand2 cardiac enhancer. ${ }^{12}$

Together, these works underscore the functional importance of IncRNA genes in fine tuning the expression of an adjacent proteincoding gene in a sequence-independent manner. The idea of sequence-independent functions of IncRNA is supported by a recent study, which showed that there is no evidence for conservation of the secondary structure of known functional IncRNA. ${ }^{13}$ Assuming that RNA-protein interaction is structure dependent, it is tempting to speculate that lack of structural conservation in IncRNA indicates that their major functions, from an evolutionary perspective, are related to their cis functions. In accordance, Kapusta and Feschotte ${ }^{14}$ proposed an evolutionary hierarchy by which IncRNA acquire functions; first. they function by regulating an adjacent gene in cis, and subsequently, they acquire trans functions (Figure 1). This is in line with higher sequence conservation observed in older mammalian-conserved IncRNA as compared with younger, humanoid-specific IncRNA. ${ }^{15}$

Analysis of human IncRNA sequences indicate that they have originated from transposable elements (TE) as $75 \%$ of human IncRNA have traces of a TE. ${ }^{16}$ It is therefore conceivable that, during evolution, insertion of a TE at a specific location could affect the expression of its adjacent genes, their promoters, and the local chromatin landscape. In cases where this insertion increased fitness, the TE element would be selected for. Indeed, regulation of the chromatin landscape occurs at several levels as well. For example, the 'ripple effect' shows that the expression of a particular gene can affect the expression of its neighbors up to $100 \mathrm{~kb}$ away. ${ }^{17}$ Together, with the fact that neighboring genes are evolutionarily linked, ${ }^{18}$ we argue that the genomic location of IncRNA may contribute significantly to their function and their birth.

Properties of IncRNA have important implications to how we study these genes. In the study of protein coding genes, the gene sequence provides the sequence of the protein product, the domains it harbors, its conservation, and rate of changes through evolution, all of which help elucidate gene function. However, in the case of IncRNA, it would seem that there is less functional information embedded in the sequence of the gene, and their major functions might be related to their genomic location. Therefore, one of the major challenges in the field of IncRNA is to be able to integrate the genomic and transcriptomic data to infer possible functions for IncRNA genes in light of the possibility that they might be noncoding and not coded.

\section{COMPETING INTEREST}

The authors declare no conflict of interest.

\section{ACKNOWLEDGEMENTS}

We wish to thank Shimon Bernstein for insightful discussion. This study was funded by research grants from the Israeli Science Foundation (ISF 1124/15) to BR.

\section{Debra Toiber ${ }^{1}$, Gabriel Leprivier ${ }^{2}$ and Barak Rotblat ${ }^{1}$ \\ ${ }^{1}$ Department of Life Sciences, Ben-Gurion University of the Negev, Beer Sheva, Israel and \\ ${ }^{2}$ Clinic for Pediatric Oncology, Hematology and Clinical Immunology, Medical Faculty, Heinrich Heine University, Dusseldorf, Germany} Correspondence: B Rotblat (rotblat@bgu.ac.il)

\section{REFERENCES}

1 Necsulea A et al. Nature 2014; 505: 635-640.

2 Tsai M-C et al. Science 2010; 329: 689-693.

3 Khalil AM et al. Proc Natl Acad Sci USA 2009; 106: 11667-11672.

4 Wang KC, Chang HY. Mol Cell 2011; 43: 904-914.

5 Xue Z et al. Mol Cell 2016; 64: 1-14.

6 Tichon A et al. Nat Commun 2016; 7: 12209.

7 Quinn JJ et al. Nat Biotechnol 2014; 32: 933-940.

8 Davidovich C et al. Nat Struct Mol Biol 2013; 20: 1250-1257.

9 Yu Y et al. Proc Natl Acad Sci 2016; 113: 11726-11731.

10 Sigova AA et al. Proc Natl Acad Sci 2013; 110: 2876-2881.

11 Engreitz JM et al. Nature 2016; 539: 452-455.

12 Anderson KM et al. Nature 2016; 539: 433-436.

13 Rivas E, Clements J, Eddy SR. Nat Methods 2016; e-pub ahead of print 7 November 2016; doi:10.1038/nmeth.4066.

14 Kapusta A, Feschotte C. Trends Genet 2014; 30: 439-452.

15 Washietl S, Kellis M, Garber M. Genome Res 2014; 24: 616-628.

16 Kapusta A et al. PLoS Genet 2013; 9: e1003470.

17 Ebisuya M et al. Nat Cell Biol 2008; 10: 1106-1113.

18 Ghanbarian AT, Hurst LD. Mol Biol Evol 2015; 32: 1748-1766.

(c) (i) This work is licensed under a Creative Commons Attribution 4.0 International License. The images or other third party material in this otherwise in the credit line; if the material is not included under the Creative Commons license, users will need to obtain permission from the license holder to reproduce the material. To view a copy of this license, visit http://creativecommons.org/licenses/ by/4.0/

(c) The Author(s) 2017 